\title{
DESCRIPCIÓN DE UNA BUENA PRÁCTICA EN EL CONTEXTO DE UN GRADO EN CIENCIAS DE LA ACTIVIDAD FÍSICA Y DEL DEPORTE: DISEÑO DE UNIDADES DE ENSEÑANZA
}

Description of a good practice in the context of a Sport Science Degree: design of teaching units

Descrição de uma boa prática no contexto da Licenciatura em Ciências do Desporto: elaboração de unidades de aprendizagem

\begin{abstract}
Este estudio se ha llevado a cabo dentro del proyecto de I+D+i: "La competencias docentes en la formación inicial del profesorado de educación física”. Convocatoria de noviembre de 2013 del Programa Estatal de Investigación, Desarrollo e Innovación Orientada a los Retos de la Sociedad, en el marco del Plan Estatal de Investigación Científica y Técnica y de Innovación 2013-2016. Referencia: EDU 2013-42024-R. Duración: 3 años (2014-2016).
\end{abstract}

\section{Carlos Gutiérrez-García}

Universidad de León, España. Teléfono: +34 987293058. Correo electrónico:

\section{cgutg@unileon.es}

\begin{abstract}
Resumen
Este trabajo describe una experiencia educativa realizada con 68 alumnos de cuarto curso del Grado en Ciencias de la Actividad Fisica y del Deporte de la Universidad de León, titulada “Trabajo grupal de diseño de una unidad de enseñanza”. Incluyó la presentación de una unidad de enseñanza por escrito, su defensa oral, la ejemplificación de una sesión y la concreción de una rúbrica. Los alumnos opinaron que fue una experiencia útil, positiva para la adquisición de competencias profesionales y satisfactoria, a pesar de su relativa dificultad. Para el profesor también fue una experiencia positiva, a pesar del gran tiempo que dedicó a ella, puesto que era el primer año en que se realizaba. También se detectaron diversos elementos de mejora sobre los que será preciso actuar en cursos sucesivos.

Palabras clave: Buenas prácticas; actividades auténticas; evaluación formativa; unidad de enseñanza; Ciencias de la Actividad Física y el Deporte
\end{abstract}




\begin{abstract}
This work describes an educational experience titled "Group work: designing a teaching unit”, which was developed with 68 students of the fourth year of the Degree in Sport Sciences of the University of León. It included the presentation of a written teaching unit, its oral defense, the exemplification of a session and the completion of a rubric. The students felt that it was a useful experience, positive for the acquisition of professional skills and satisfactory, despite its relative difficulty. For the teacher it was also a positive experience, despite the great time he devoted to it, since it was the first year it was implemented. A number of elements of improvement were also detected on which it will be necessary to act during the following courses.
\end{abstract}

Keywords: Good practices; authentic activities; formative assessment; teaching unit; Sports Science

\title{
Resumo
}

Este trabalho descreve uma experiência educativa titulada "Trabalho grupal: elaboração de uma unidade de ensino”, que foi desenvolvida com 68 alunos do quarto ano da Licenciatura em Ciências do Desporto da Universidade de León. Incluía a apresentação de uma unidade de ensino escrita, a sua defesa oral, a exemplificação de uma sessão e a concreção de uma rubrica. Os alunos consideraram que a experiência foi útil, positiva para a aquisição de competências profissionais e satisfatória, apesar da sua relativa dificuldade. Para o professor também foi uma experiência positiva, apesar do grande tempo que dedicou a ela, uma vez que foi o primeiro ano em que se realizaba. Foram detectados também alguns elementos de melhoria nos quais será necessário agir durante os cursos seguintes.

Palavras-chave: Boas práticas; actividade autêntica; avaliação formativa; unidade de aprendizagem; Ciências do Desporto

\section{Introducción}

El objetivo del presente trabajo es describir el diseño, desarrollo y evaluación de una experiencia educativa en el contexto universitario titulada "Trabajo grupal de diseño de una unidad de enseñanza”. En ella cobran fuerza conceptos como actividad / evaluación 
auténtica, evaluación para el aprendizaje, evaluación formativa o evaluación participativa (López-Pastor, 2017; Vallejo \& Molina, 2014), en el convencimiento, cada vez más respaldado científicamente, de que se trata de elementos clave para favorecer el desarrollo de competencias por parte del alumnado.

\section{Contextualización}

La experiencia se realizó en la asignatura "Diseño y análisis de la enseñanza de la actividad física” (DIS), cuyo objetivo principal es capacitar al alumnado para elaborar y evaluar programas de enseñanza de actividad física. DIS es una asignatura obligatoria de cuarto curso del Grado en Ciencias de la Actividad Física y el Deporte de la Universidad de León, que tiene asignados un total de seis créditos (dos teóricos y cuatro prácticos) y se imparte durante el primer semestre del curso. Durante el curso académico en el que se ha desarrollado la experiencia (2016-2017), hubo 74 alumnos matriculados (13 alumnas y 61 alumnos), de los cuales 68 (91,9\%) realizaron la experiencia planteada (trabajo grupal y actividades asociadas), mientras que el resto optó por presentarse únicamente al examen final de la asignatura. Estos últimos alumnos eran fundamentalmente alumnos repetidores. Los créditos teóricos se impartieron en gran grupo y para los prácticos se contó con dos grupos. La totalidad de la asignatura fue impartida por un único profesor, el autor del presente trabajo. Este fue el primer año en que la experiencia se puso totalmente en práctica, aunque el profesor ya tenía experiencia previa en la asignatura y en buenas prácticas y evaluación formativa.

\section{Diseño y desarrollo}

La experiencia o buena práctica "Trabajo grupal de diseño de una unidad de enseñanza" es una propuesta compleja a través de la cual se tratan de integrar de forma práctica y auténtica la mayor parte de contenidos de la asignatura DIS. Se basa en la realización de un trabajo grupal (grupos de 4-6 alumnos) consistente en una unidad de enseñanza (UE) completa adaptada a un contexto y a una programación concreta de actividad física. Los alumnos pueden elegir, por ejemplo, centros de ocio y tiempo libre, actividades extraescolares, centros deportivos, organizaciones sociales, cárceles, centros de refugiados, etc., pero no educación formal, ya que este ámbito corresponde al máster de formación del profesorado. La elaboración de la UE incluye: (1) la entrega del trabajo 
impreso, junto con una rúbrica de evaluación cumplimentada (autoevaluación del grupo); (2) la presentación oral del mismo en formato PechaKucha (PK), de forma individual; (3) la elaboración de uno de los apartados de la rúbrica (cada grupo un apartado diferente); y (4) la selección de una sesión de la UE para ser realizada durante una sesión práctica de la asignatura. De forma esquemática, la experiencia puede representarse muestra la Figura 1

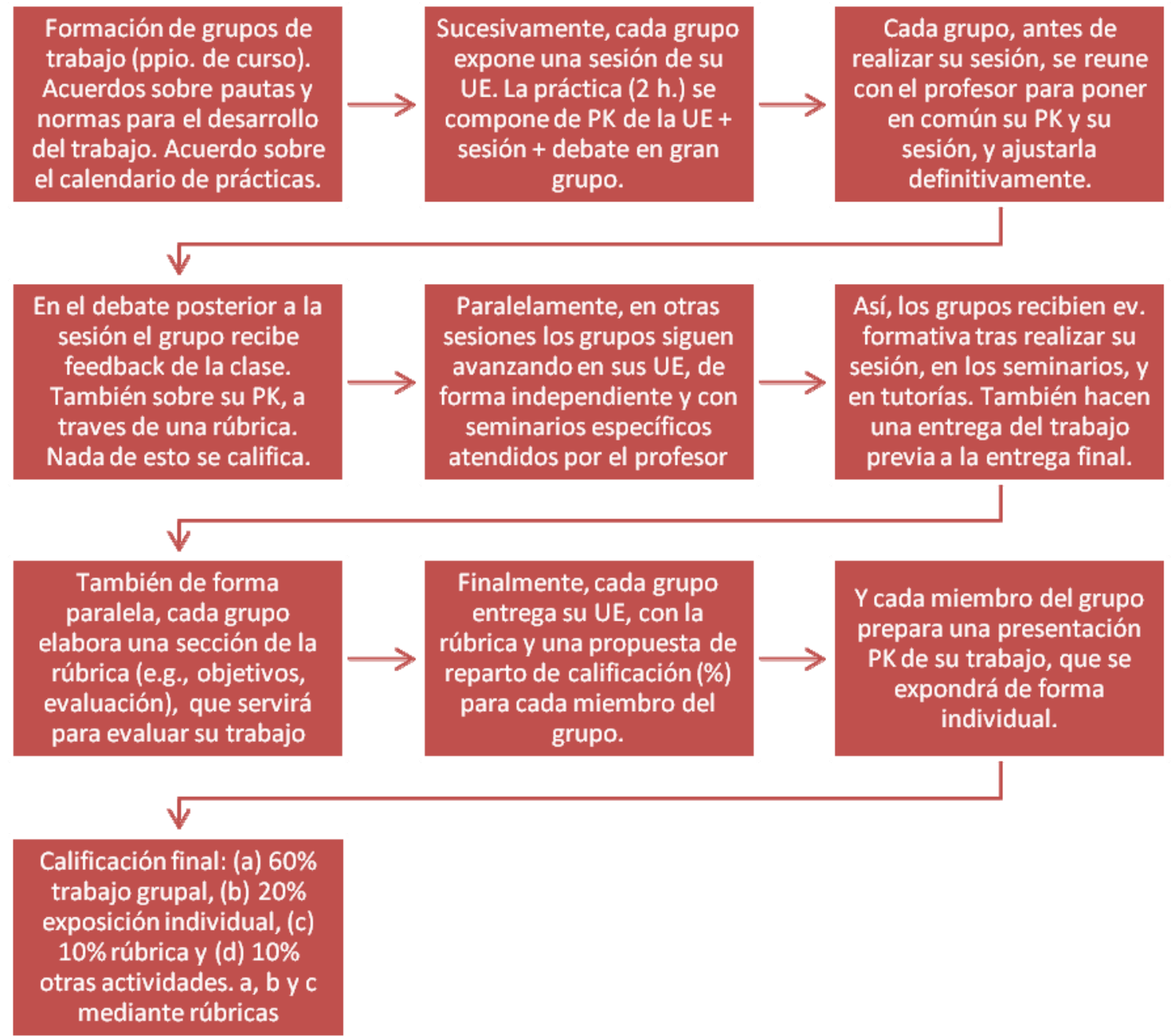

Nota: UE = Unidad de enseñanza; PK = PechaKucha.

Figura 1.

Esquema de la experiencia "Trabajo grupal de diseño de una unidad de enseñanza”.

La experiencia se desarrolló de forma bastante ajustada a lo diseñado y acordado inicialmente con el grupo, y únicamente fueron necesarias adaptaciones sobre la temporalización para atender situaciones sobrevenidas. La totalidad de los grupos cumplieron sus compromisos en tiempo y forma, haciendo un importante uso de las tutorías, particularmente a nivel de grupos de trabajo. Como principal carencia, se Descripción de una buena práctica en el contexto de un Grado en Ciencias de la Actividad Física y del Deporte: diseño de unidades de enseñanza 
evidenciaron desajustes entre el diseño de la rúbrica para el trabajo grupal y su aplicación para la elaboración del trabajo, ya que al ser procesos paralelos no se pudo utilizar realmente hasta el final de la asignatura.

\section{Evaluación}

Para la evaluación de la experiencia, se tomó como referencia el Cuestionario sobre la experiencia de "buena práctica” y su evaluación, de la Red de Evaluación Formativa y Compartida en Educación Superior (Romero, Asún, \& Chivite, 2016). Como muestra la Figura 2, los alumnos consideraron que la experiencia fue bastante útil y positiva para la adquisición de competencias profesionales. También les resultó bastante satisfactoria, a pesar que su dificultad, que fue valorada entre medianamente y bastante difícil. Con todo, estos datos han de ser tomados con cautela ya que únicamente 38 alumnos (51,35\% del total) respondieron el cuestionario, realizado on-line de forma voluntaria.

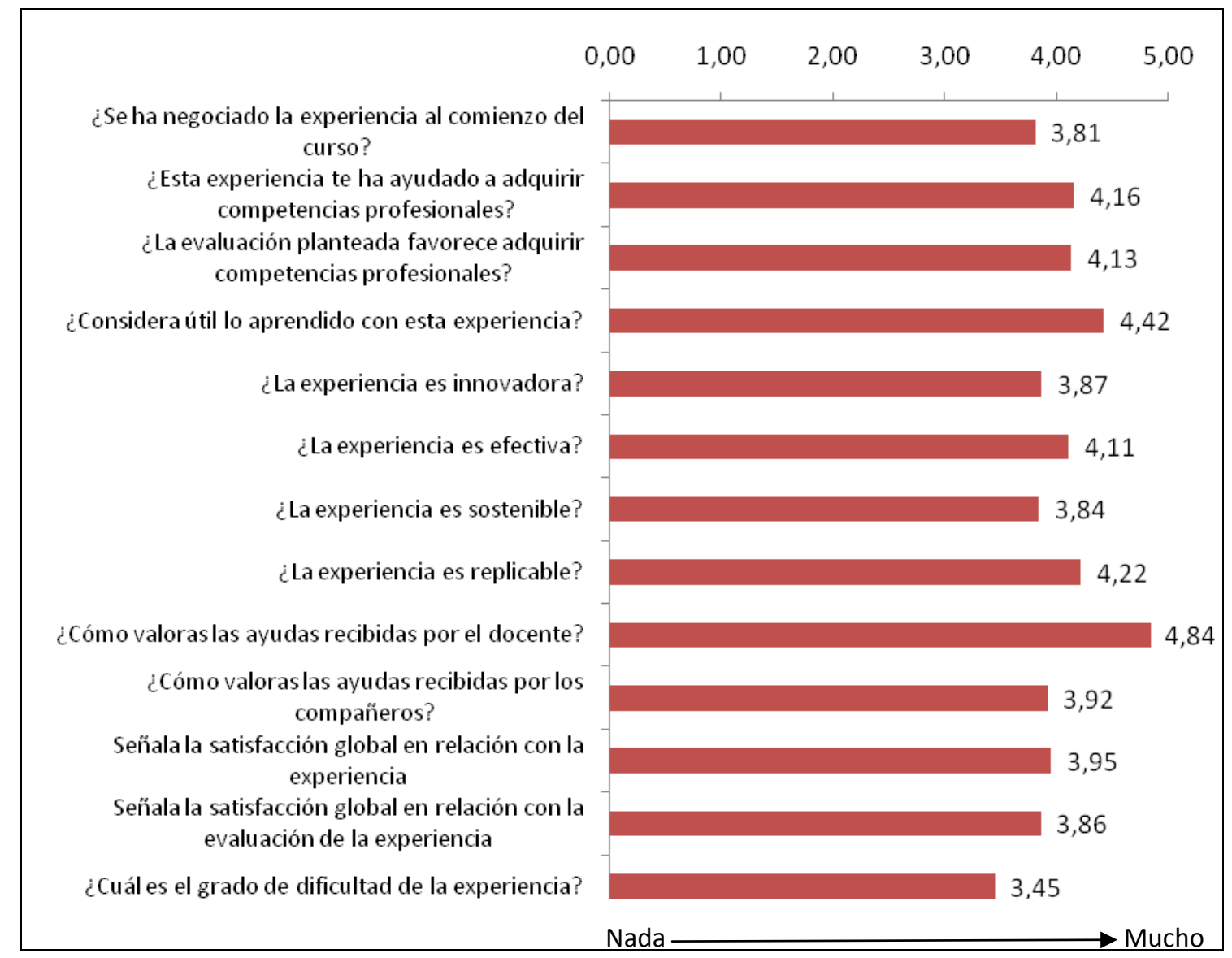

Figura 2.

Valoraciones de la experiencia por los alumnos $(n=38)$.

Descripción de una buena práctica en el contexto de un Grado en Ciencias de la Actividad Física y del Deporte: diseño de unidades de enseñanza 
Como profesor, también valoro la experiencia positivamente, en sintonía con los alumnos. Me supuso un gran trabajo, al ser el primer año de realización de la experiencia, que con toda seguridad será menor en cursos sucesivos. Los principales aspectos a destacar son: (1) la implicación de los alumnos en el proceso; (2) el carácter formativo de la buena práctica; (3) su carácter auténtico; y (4) el desarrollo de procesos de evaluación transparentes. Como aspectos a mejorar destacan: (1) disponer de la rúbrica del trabajo desde principio de curso; y (2) mejorar la implicación de los alumnos en las sesiones prácticas.

Por último, las calificaciones finales de la asignatura se muestran en la Tabla 1. El 80,9\% de los alumnos que realizó la experiencia superó la asignatura en la primera convocatoria, y únicamente dos (2,9\%) de los alumnos restantes no lograron hacerlo en la segunda convocatoria. Solo un alumno de los seis que optaron por la opción de examen final superó la asignatura (16,7\%)

Tabla 1.

Calificaciones finales de la asignatura de los alumnos que realizaron la experiencia $(n=68)$

\begin{tabular}{lcc}
\hline Calificación & Primera convocatoria (enero) & Segunda convocatoria (febrero) \\
\hline Matrícula de Honor & $4(5,9 \%)$ & $0(0 \%)$ \\
Sobresaliente & $3(4,4 \%)$ & $0(0 \%)$ \\
Notable & $19(27,9 \%)$ & $10(14,7 \%)$ \\
Aprobado & $29(42,6 \%)$ & $1(1,5 \%)$ \\
Suspenso & $13(19,1 \%)$ & $2(2,9 \%)$ \\
No presentado & $0(0 \%)$ & $0(0 \%)$ \\
\hline
\end{tabular}

\section{Conclusiones}

Los resultados durante este primer año han sido satisfactorios, aunque existen elementos que deben mejorarse para el próximo año. Además de los señalados, es preciso mejorar la recogida de datos respecto a los cuestionarios para favorecer una máxima representatividad de los resultados. Es deseable que para el próximo curso exista una coordinación aún mejor de todos los procesos de la experiencia, que permita optimizar el tiempo dedicado a la asignatura, tanto por parte de los alumnos como del profesor.

\section{Referencias}

López-Pastor, V.M. (2017). Evaluación formativa y compartida: evaluar para aprender y la implicación del alumnado en los procesos de evaluación y aprendizaje. En V.M. López-Pastor \& Á. Pérez-Pueyo (Eds.), Evaluación formativa y compartida 
en educación: experiencias de éxito en todas las etapas educativas (pp. 34-68).

León: Universidad de León. Recuperado de https://buleria.unileon.es/handle/10612/5999

Romero, M.R., Asún, S., \& Chivite, M. (2016). La autoevaluación en expresión corporal en formación inicial del profesorado de educación física: un ejemplo de buena práctica. Retos: nuevas tendencias en educación física, deporte y recreación, (29), 236-241.

Vallejo, M., \& Molina, J. (2014). La evaluación auténtica de los procesos educativos. Revista Iberoamericana de Educación, (64), 11-25. 